\title{
Linear Equalization Combined with Multiple Symbol Decision Feedback Detection for Differential Space-Time Modulation
}

\author{
Genyuan Wang \\ Department of Electrical and Computer Engineering, University of Delaware, Newark, DE 19716, USA \\ Email:gwang@ee.udel.edu
}

\author{
Aijun Song \\ Department of Electrical and Computer Engineering, University of Delaware, Newark, DE 19716, USA \\ Email:song@ee.udel.edu
}

\section{Xiang-Gen Xia}

Department of Electrical and Computer Engineering, University of Delaware, Newark, DE 19716, USA

Email:xxia@ee.udel.edu

Received 30 May 2001 and in revised form 19 February 2002

\begin{abstract}
Differential space-time modulation (DSTM) for multiple antenna communication systems has been recently proposed for frequency-nonselective fading channels. In broadband multirate systems, frequency-selective fading may occur. In this paper, the DSTM in frequency-selective fading channels is considered. A linear equalizer for the DSTM is proposed over frequency-selective fading channels. Furthermore, the multiple symbol decision feedback detection is used to improve the performance of the linear equalizer.
\end{abstract}

Keywords and phrases: differential space-time modulation, transmit diversity, decision feedback detection, MIMO, linear equalization, RLS.

\section{INTRODUCTION}

Differential space-time modulation (DSTM) has been recently proposed by Hochwald and Sweldens [1], Hughes [2], and Tarokh and Jafarkhani [3] for multiple antenna communication systems, where the channel information is not known to the transmitters or receivers. Several unitary spacetime codes for the DSTM have been proposed in $[1,2]$ of group codes, in $[2,4]$ of quaternion codes, and in [5] of fixedpoint free group codes, and in [6] of parametric codes. The $2 \times 2$ parametric codes obtained in [6] for two transmit antennas have the largest possible diversity product of size five and the largest known diversity product of size sixteen, which will be used in the simulations in this paper later.

Space-time coded multiple antenna systems can be casted as multiple input and multiple output (MIMO) systems. The recent studies of DSTM in $[1,2,3]$ are on frequencynonselective fading channels where MIMO channel matrices do not have memory. However, in broadband multirate systems, frequency-selective fading and frequency-nonselective fading may co-exist. In this paper, we are interested in the
DSTM system when frequency-selective fading occurs. An equalizer combined with the differential detection is proposed by exploiting the structure of the DSTM and its differential detection. The equalizer is to minimize the detection errors. In such an equalizer, not only the channels are of matrix form but also the input signals and the output signals are of matrix form too. Similar to the existing MIMO equalization techniques in the literature, see for example $[7,8]$, the least squares (LS) solution and an adaptive RLS algorithm are derived. As a remark, equalizations of space-time trellis coded multi-antenna systems over frequency-selective fading channels have been considered in $[9,10]$.

The DSTM can be treated as a generalization of differential phase shift keying (DPSK). Thus, its differential detection scheme is similar to that of DPSK [1]. Decision feedback detection schemes have been used to improve the performance for the DPSK systems in [11]. In this paper, we also generalize this idea to the equalizer for the DSTM system by incorporating the multiple symbol decision feedback detection in the linear equalizer.

This paper is organized as follows. The differential space- 


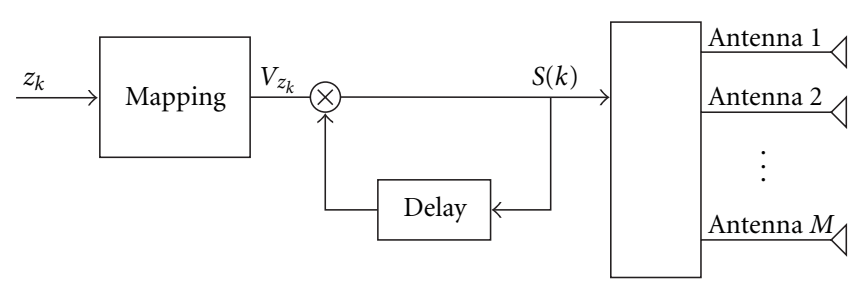

FIGURE 1: The transmission scheme of differential space-time modulation with multiple antennas.

time modulation is briefly reviewed with the frequencyselective fading channel model in Section 2. The equalization scheme is presented under the LS criterion and the equalizer combined with the multiple symbol decision feedback detection is presented in Section 3. In Section 4, the RLS algorithm is derived. Simulation results and conclusions are given in Sections 5 and 6 , respectively.

\section{DIFFERENTIAL SPACE-TIME MODULATION AND SIGNAL MODEL}

Figure 1 shows the transmission scheme of differential spacetime modulation. Suppose there are $M$ transmit, $N$ receive antennas in a frequency-selective fading environment. The transmission rate is $R_{t}$ bits per channel use.

An information sequence is grouped as $z_{k}$ and then mapped to $V_{z_{k}}, V_{z_{k}} \in\left\{V_{l}, l=0,1, \ldots, L-1\right\}$, a space-time code, where $L=2^{M R_{t}}$ and $L$ is the signal constellation size. Using the differential modulation technique (similar to the single antenna system), the transmit signal of the $k$ th block is

$$
S(k)=S(k-1) V_{z_{k}}
$$

where

$$
S(k)=\left[\begin{array}{cccc}
s_{1, k M} & s_{1, k M+1} & \cdots & s_{1,(k+1) M-1} \\
s_{2, k M} & s_{2, k M+1} & \cdots & s_{2,(k+1) M-1} \\
\vdots & \vdots & \vdots & \vdots \\
s_{M, k M} & s_{M, k M+1} & \cdots & s_{M,(k+1) M-1}
\end{array}\right],
$$

$s_{m, t}$ is the transmitted signal on the $m$ th transmit antenna at time $t$ and $S(0)$ is the $M \times M$ identity matrix. Since $V_{z_{k}}$ is a unitary matrix, $S(k)$ is also unitary. Thus, the mean signal power at time $t$ is always unit, that is,

$$
\mathbf{E} \sum_{m=1}^{M}\left|s_{m, t}\right|^{2}=1
$$

On the $n$th receive antenna at time $t$, the received signal is

$$
x_{n, t}=\sqrt{\rho} \sum_{m=1}^{M} \sum_{d=0}^{L_{h}-1} h_{n, m}(t, d) s_{m, t-d}+w_{n, t},
$$

where $h_{n, m}(t, d)$ is the $d$ th tap coefficient of the channel between the $m$ th transmit and the $n$th receive antennas at time $t, w_{n, t}$ is the additive noise on the $n$th receive antenna at time $t, L_{h}$ is the largest delay spread of all channels, and $\rho$ is the signal-to-noise ratio (SNR) on the receive antennas. For the energy normalization purpose, the channels are passive as in [9], that is,

$$
\sum_{d=0}^{L_{h}-1}\left|h_{n, m}(t, d)\right|^{2}=1
$$

It is assumed that $w_{n, t}$ is complex Gaussian white noise with zero mean and unit variance, that is, $w_{n, t} \sim C N(0,1)$ and $w_{n, t}$ is independent of each other with respect to both $n$ and $t$. As in $[1,2,3,4,5,6], h_{n, m}(t, d)$ is assumed independent of each other with respect to $m$ and to $n$ but correlated with respect to $t$. When the fading is slow, the channel coefficients can be taken as constant over a period of time, coherence time. Thus $h_{n, m}(t, d)$ can be represented by $h_{n, m}(d)$ within the coherence time. Therefore, (4) can be written in the following matrix form:

$$
X(k)=\sqrt{\rho} \sum_{d=0}^{L_{h}-1} H_{d} S(k-d)+W(k)
$$

where

$$
\begin{gathered}
X(k)=\left[\begin{array}{cccc}
x_{1, k M} & x_{1, k M+1} & \cdots & x_{1,(k+1) M-1} \\
x_{2, k M} & x_{2, k M+1} & \cdots & x_{2,(k+1) M-1} \\
\vdots & \vdots & \vdots & \vdots \\
x_{N, k M} & x_{N, k M+1} & \cdots & x_{N,(k+1) M-1}
\end{array}\right], \\
H_{d}=\left[\begin{array}{cccc}
h_{1,1}(d) & h_{1,2}(d) & \cdots & h_{1, M}(d) \\
h_{2,1}(d) & h_{2,2}(d) & \cdots & x_{2, M}(d) \\
\vdots & \vdots & \vdots & \vdots \\
h_{N, 1}(d) & h_{N, 1}(d) & \cdots & h_{N, M}(d)
\end{array}\right],
\end{gathered}
$$

and $W(k)$ is the $M \times N$ noise matrix on the receive antennas at the $k$ th block, which is from $w_{n, t}$.

In $[1,2,3,4,5,6]$, delay spread $L_{h}=1$ is assumed. In this case, (6) becomes

$$
X(k)=\sqrt{\rho} H S(k)+W(k),
$$

where $H=\left(h_{n, m}\right)_{N \times M}$.

When channel matrix is approximately constant during the transmission of two blocks of data, (8) can be rewritten as

$$
\begin{aligned}
X(k) & =\sqrt{\rho} H S(k-1) V_{z_{k}}+W(k) \\
& =(X(k-1)-W(k-1)) V_{z_{k}}+W(k) \\
& =X(k-1) V_{z_{k}}+W^{\prime}(k),
\end{aligned}
$$

where $W^{\prime}(k)=W(k)-W(k-1) V_{z_{k}}$. As $V_{z_{k}}$ is unitary, the additive noise $W^{\prime}(k)$ is still independent Gaussian. Therefore, the maximum-likelihood detector $[1,2]$ for $(11)$ is

$$
V_{z_{k}}=\arg \min _{l=0,1, \ldots, L-1}\left\|X(k)-X(k-1) V_{z_{l}}\right\|
$$


where $\|A\|$ is the Frobenius norm of $A=\left(a_{m n}\right)_{M \times N}$, defined as

$$
\|A\|=\sqrt{\operatorname{Tr}\left(A^{H} A\right)}=\left(\sum_{m=1}^{M} \sum_{n=1}^{N}\left|a_{m n}\right|^{2}\right)^{1 / 2} .
$$

\section{EQUALIZATION OF DIFFERENTIAL SPACE-TIME MODULATED SYSTEM}

In broadband multirate wireless communication links, the assumption of delay spread $L_{h}=1$ does not always hold. When multipaths exist, equalization techniques are needed to compensate the intersymbol interference (ISI). In the single antenna case, the linear equalizer works as a filter to suppress the ISI term. The scalar coefficients of the equalizer are the parameters of the filter. In the multiple transmit antenna system, such coefficients are matrices. The input and the output are also matrices. The structure of the equalization is shown in Figure 2.

Therefore, the output of such equalizer is

$$
\begin{aligned}
Q(k) & =\sum_{v=0}^{L_{e}-1} \underline{c}_{v} X(k-v) \\
& =G_{k_{0}} S\left(k-k_{0}\right)+\sum_{v=0, v \neq k_{0}}^{L_{h}+L_{e}-2} G_{v} S(k-v)+\sum_{v=0}^{L_{e}-1} \underline{c}_{v} W(k-v),
\end{aligned}
$$

where $\underline{c}_{v}, v=0,1, \ldots, L_{e}-1$, are $N \times N$ coefficient matrices for equalization, and $L_{e}$ is the length of the equalizer,

$$
G_{v}=\sum_{\mu=0}^{L_{e}-1} \underline{c}_{\mu} H(v-\mu)
$$

is the equivalent linear transform of the cascade of the channel and the equalizer, and $k_{0}$ is the overall delay caused by the linear transform $G_{v}$.

After equalization, the ISI item, that is, the second item in (15), is compensated. So

$$
Q(k)=G_{k_{0}} S\left(k-k_{0}\right)+\sum_{v=0}^{L_{e}-1} \underline{c}_{v} W(k-v) .
$$

Therefore, (12) can be applied to (17). That is,

$$
\hat{V}_{z_{k-k_{0}}}=\arg \min _{l=0,1, \ldots, L-1}\left\|Q(k)-Q(k-1) V_{z_{l}}\right\| .
$$

As in the DPSK with single antenna $[11,12], Q(k-1)$ can be substituted by

$$
Q_{\mathrm{ref}}(k)=\frac{1}{L_{n}-1} \sum_{j=1}^{L_{n}-1} Q(k-j) \prod_{i=j-1}^{1} \hat{V}_{z_{k-k_{0}-i}},
$$

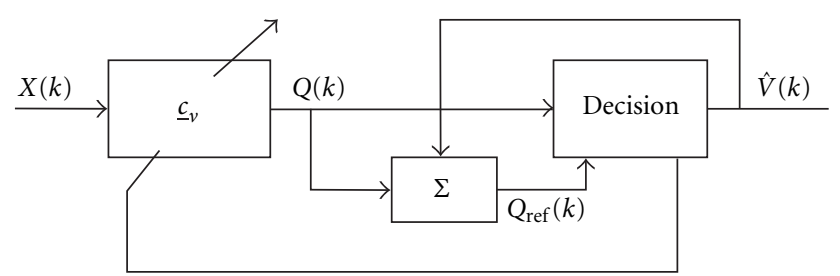

FIGURE 2: Block diagram of receiver combining linear equalization with multiple symbol feedback detection for differential space-time modulation.

where feedback order $L_{n}$ is the number of previous equalization outputs used in the decision. If $L_{n}=2, Q_{\mathrm{ref}}(k)=$ $Q(k-1)$. In (19), each item in the summation is the estimation of $Q(k-1)$. $Q_{\text {ref }}(k)$ is the average of $L_{n}-1$ estimated values of $Q(k-1)$ and therefore less noisy. Therefore, when $L_{n}>2$, performance improvement can be obtained. Thus the detector turns into

$$
\hat{V}_{z_{k-k_{0}}}=\arg \min _{l=0,1, \ldots, L-1}\left\|Q(k)-Q_{\text {ref }}(k) V_{z_{l}}\right\| .
$$

The equalizer is to minimize the error $\mathbf{e}(k)=Q(k)-$ $Q_{\text {ref }}(k) V_{z_{k}}$. In the system, the cost function is defined as in [13],

$$
\psi(k)=\sum_{\tau=1}^{k} \lambda^{k-\tau}\|\mathbf{e}(\tau)\|^{2}
$$

where $\lambda$ is the forgetting factor. Under the LS criterion, the equalization coefficient matrices $\underline{c}_{v}, v=0,1, \ldots, L_{e}-1$ can be obtained. With the obtained equalizer coefficients, the detector (20) can be applied with the equalization output $Q(k-j)$, $j=0,1, \ldots, L_{n}-1$.

\section{RLS ALGORITHM FOR DIFFERENTIAL SPACE-TIME MODULATED SYSTEM}

In the forms of (14), (15), and (16) in Section 3, the channels, the input and output signals are of matrix forms. Similar to the existing equalization techniques for MIMO systems $[7,8,14]$, we can derive the LS solution and the RLS solution for differential space-time modulated systems as follows.

Let $c_{v}^{i l}, x^{i l}(k)$ denote $i, j$ th element of matrix $\underline{c}_{v}$ and $X(k)$, respectively, then

$$
\begin{aligned}
\underline{c}^{i l} & =\left[\begin{array}{llll}
c_{0}^{i l} & c_{1}^{i l} & \cdots & c_{L_{e}-1}^{i l}
\end{array}\right]^{H}, \\
\underline{x}^{i l}(k) & =\left[\begin{array}{llll}
x^{i l}(k) & x^{i l}(k-1) & \cdots & x^{l j}\left(k-L_{e}+1\right)
\end{array}\right]^{T},
\end{aligned}
$$

where the superscript $H$ denotes the complex conjugate transpose, and $T$ denotes the transpose. Equation (14) can be written in matrix form

$$
Q(k)=C^{H} R(k),
$$


where

$$
\begin{aligned}
C & =\left[\begin{array}{cccc}
\underline{c}^{11} & \underline{c}^{21} & \cdots & \underline{c}^{N 1} \\
\underline{c}^{12} & \underline{c}^{22} & \cdots & \underline{c}^{N 2} \\
\vdots & \vdots & \vdots & \vdots \\
\underline{c}^{1 N} & \underline{c}^{2 N} & \cdots & \underline{c}^{N N}
\end{array}\right], \\
R(k) & =\left[\begin{array}{cccc}
\underline{x}^{11}(k) & \underline{x}^{12}(k) & \cdots & \underline{x}^{1 M}(k) \\
\underline{x}^{21}(k) & \underline{x}^{22}(k) & \cdots & \underline{x}^{2 M}(k) \\
\vdots & \vdots & \vdots & \vdots \\
\underline{x}^{N 1}(k) & \underline{x}^{N 2}(k) & \cdots & \underline{x}^{N M}(k)
\end{array}\right] .
\end{aligned}
$$

In addition,

$$
D(k)=Q_{\text {ref }}(k) \hat{V}_{z_{k-k_{0}}} .
$$

Therefore $C$, which minimizes $\psi(k)$, can be obtained by differentiating $\psi(k)$ in $(21)$ and setting the result to be the zero matrix, that is,

$$
\phi(k) C=\varphi(k),
$$

where

$$
\begin{aligned}
& \phi(k)=\sum_{\tau=1}^{k} \lambda^{k-\tau} R(\tau) R^{H}(\tau), \\
& \varphi(k)=\sum_{\tau=1}^{k} \lambda^{k-\tau} R(\tau) D^{H}(\tau) .
\end{aligned}
$$

Note that $R(\tau)$ and $D(\tau)$ are matrices rather than vectors in conventional normal equation [13]. Using the matrix inversion lemma [13], an iterative algorithm for (26) can be obtained. Hence, the RLS algorithm for the space-time modulation with multiple antennas is as follows:

$$
\begin{aligned}
P(0)= & \sigma^{-1} I_{L_{e} \times N}, \quad \sigma=0.004, \\
\Gamma(k)= & \lambda^{-1} P(k-1) R(k) \\
& \times\left(I_{M}+\lambda^{-1} R^{H}(k) P(k-1) R(k)\right)^{-1}, \\
\xi(k)= & D(k)-C^{H}(k) R(k), \\
C(k)= & C(k-1)+\Gamma(k) \xi^{H}(k), \\
P(k)= & \lambda^{-1} P(k-1)-\lambda^{-1} \Gamma(k) R^{H}(k) P(k-1),
\end{aligned}
$$

where $I_{v}$ is the $v \times v$ identity matrix and $C(k)$ denotes the value of $C$ after $k$ iterations. If $M=N=1$, this is just the RLS algorithm in [13]. From (25), (23), (19), (31), and (32), $C(0)$ should have a nonzero value. In the following simulations, all the elements of $\underline{c}_{k 0}$ are set to 0.1 , that is, $\underline{c}_{k 0}^{i j}=0.1$, $i, j=1,2, \ldots, N$. For fixed channels, $\lambda$ can be set to 1 and $\hat{V}_{z_{k-k_{0}}}$ are assumed known in the training. After the training, the detector in (20) is applied. $\hat{V}_{z_{k-k_{0}}}$ is the previous detection result, to which the multiple symbol decision feedback detection refers. For the fading channels, $\lambda$ can be set to a value less than 1 . Thus, the receiver can track the variation of the channels.

\section{SIMULATION RESULTS}

In this section, the performance of the equalization scheme for differential space-time modulated system is shown with simulations. In the following simulations, parametric spacetime code of size 16 in [6] is adopted. For $l=0,1, \ldots, 15$,

$$
\begin{aligned}
V_{l}= & {\left[\begin{array}{cc}
\exp \left(\frac{j l \pi}{8}\right) & 0 \\
0 & \exp \left(\frac{j 3 l \pi}{8}\right)
\end{array}\right] } \\
& \times\left[\begin{array}{cc}
\cos \left(\frac{l \pi}{2}\right) & \sin \left(\frac{l \pi}{2}\right) \\
-\sin \left(\frac{l \pi}{2}\right) & \cos \left(\frac{l \pi}{2}\right)
\end{array}\right] \\
& \times\left[\begin{array}{cc}
\exp \left(\frac{j l \pi}{4}\right) & 0 \\
0 & \exp \left(\frac{-j l \pi}{4}\right)
\end{array}\right] .
\end{aligned}
$$

This code has the best known product diversity in the literature of the same size, which is $2^{1 / 4} / 2$ following the notation in [1]. Although this code itself is not a group, it is a subset of a group of size 32 [6]. Using this space-time code, the number of transmit antennas is $M=2$ and the transmission data rate is $R_{t}=2$. The number of receive antennas is set as 2, that is, $N=2$. The channel delay spread $L_{h}=2$ is used throughout the simulations.

The simulation results are given under two cases, fixed channels and fading channels. In the fixed channel case, the two taps of a channel have equal power as the channels studied in [9]. Figure 3 shows the learning curve of the RLS algorithm for differential space-time modulation. The length of equalizer $L_{e}=3$. An information sequence of about 300 symbols known to the receiver is used in the training. $J(k)=$ $\mathbf{E}\|\mathbf{e}(k)\|^{2}$. The curves are drawn from the average over 1000 training processes. The forgetting factor $\lambda=1.0$. The overall delay $k_{0}$ is set to zero. Though the convergence processes do not speed up remarkably, the errors under concern decrease as the increase of $L_{n}$, the feedback order.

The performances of different receivers are shown in Figure 4 . Because the energy of first taps and that of the second taps are the same, the ratio of the desired signals to the interference part is $0 \mathrm{~dB}$ when no equalization techniques is used. In this case, the performance is not good (BLER is above $10^{-1}$ ) whatever SNR is. Thus, this case is not shown in Figure 4. From Figure 4 one can see that the increase of the feedback order $L_{n}$ improves the performance, especially in the high SNR. However, the increase of $L_{n}$ also means the increase of the computational complexity.

The performances of the receive scheme over fading channels are shown in Figure 5. All the channel coefficients, independent of each other, are generated by Jakes's model [15]. The auto-correlation of a fading channel of $t$ time samples apart is $J_{0}\left(2 \pi f_{d} T_{s} t\right)$, where $J_{0}$ is the zero-order Bessel function of the first kind, $f_{d} T_{s}$ is the fading rate. In GSM 


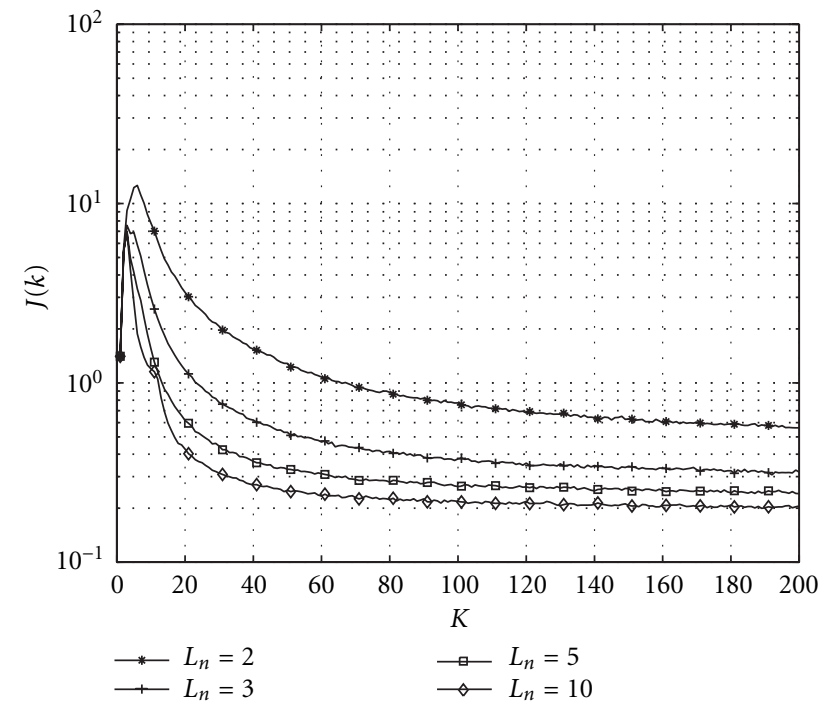

FIGURE 3: Learning curves of the RLS algorithm for space-time modulation with different $L_{n}$.

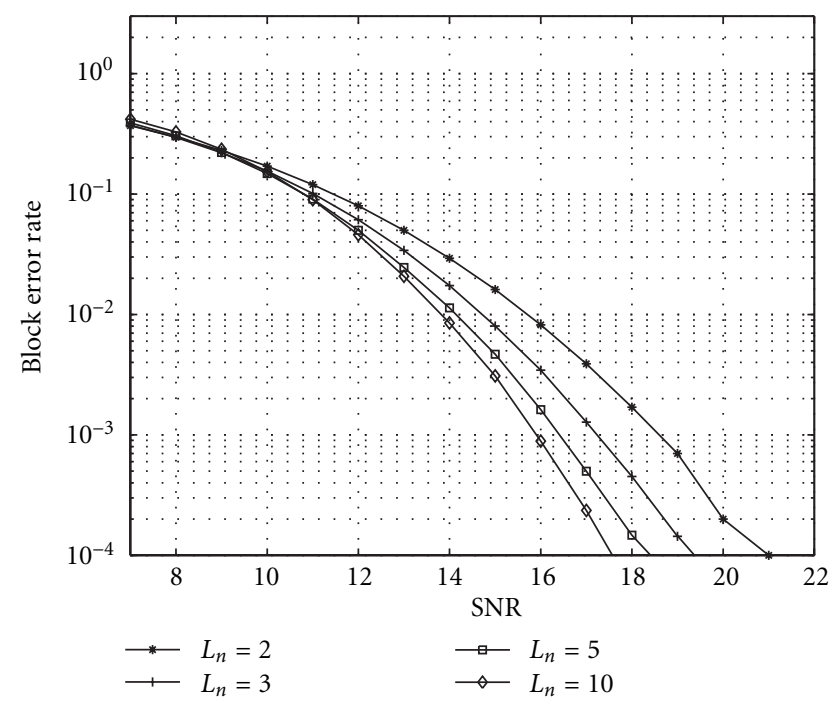

FIGURE 4: The block error rate of space-time modulated system under fixed ISI channels.

system, the carrier frequency is $900 \mathrm{MHz}$. If a basic GSM EDGE slot structure with symbol rate of $2.71 \times 10^{5} \mathrm{symbols} / \mathrm{s}$ is used and the speed of mobile station is $3 \mathrm{~km} / \mathrm{h}$ (TU3), the fading rate $f_{d} T_{s}$ is $10^{-5}$, which is assumed in the simulation. The average energy for the first taps of the channels is $9 / 10$ of the total energy. The length of the equalizer $L_{e}=3$. Before the fading applied, 800 symbols are used in training. After training, $\lambda$ is set as 0.98 . The curve marked by $\circ$ in Figure 5 is the performance without equalization, which has an error floor around BLER of $10^{-1}$. It is shown that the receiver with $L_{n}>2$ still has a better performance over the receiver with $L_{n}=2$. However, the receiver with $L_{n}=10$ has approximately the same BLER with the receiver with $L_{n}=5$. The reason lies

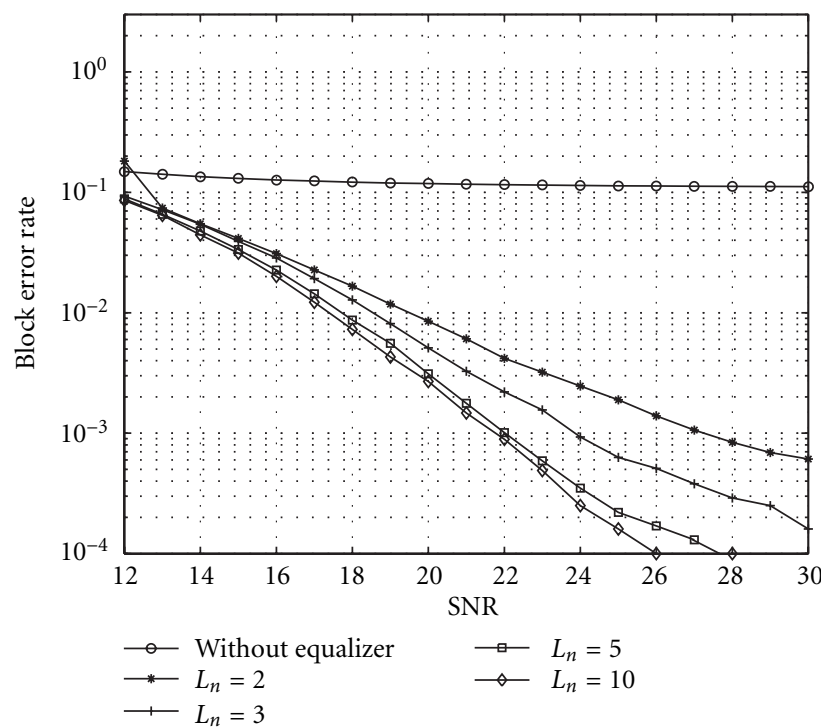

FIGURE 5: The block error rate of space-time modulation with equalization under frequency selective fading environment, $f_{d} T_{s}=$ $10^{-5}$.

is that (18) does not give a better estimation of $Q(k-1)$ due to the fading of the channel.

\section{CONCLUSIONS}

In the paper, an equalizer combined with differential detection was proposed for the differential space-time modulated system over frequency selective fading channels. By applying the LS criterion on the detection error, the RLS algorithm was derived. Also the multiple symbol decision feedback detection was incorporated to improve the performance of the combined equalizer and detector. The performance was illustrated by simulations. After this paper was done, we found a related and independent work [16].

\section{ACKNOWLEDGMENTS}

This work was supported in part by the Air Force Office of Scientific Research (AFOSR) under Grant F49620-00-1-0086 and the National Science Foundation (NSF) Grants MIP9703377 and CCR-0097240.

\section{REFERENCES}

[1] B. M. Hochwald and W. Sweldens, "Differential unitary spacetime modulation," IEEE Trans. Communications, vol. 48, no. 12, pp. 2041-2052, 2000.

[2] B. L. Hughes, "Differential space-time modulation," IEEE Transactions on Information Theory, vol. 46, no. 7, pp. 25672578,2000

[3] V. Tarokh and H. Jafarkhani, "A differential detection scheme for transmit diversity," IEEE Journal on Selected Areas in Communications, vol. 18, no. 7, pp. 1169-1174, 2000.

[4] B. L. Hughes, "Optimal space-time constellations from groups," submittedd to IEEE Transactions on Information Theory, 2000. 
[5] A. Shokrollahi, B. Hassibi, B. M. Hochwald, and W. Sweldens, "Representation theory for high-rate multiple-antenna code design," IEEE Transactions on Information Theory, vol. 47, no. 6, pp. 2335-2367, 2000.

[6] X.-B. Liang and X.-G. Xia, "Unitary signal constellations for differential space-time modulation with two transmit antennas: Parametric codes, optimal designs, and bounds," to appear in IEEE Transactions on Information Theory.

[7] N. Al-Dhahir and A. H. Sayed, "The finite-length multi-input multi-output MMSE-DFE,” IEEE Trans. Signal Processing, vol. 48, no. 10, pp. 2921-2936, 2000.

[8] A. Duel-Hallen, "Equalizers for multiple input/output channels and PAM systems with cyclostationary input sequences," IEEE Journal on Selected Areas in Communications, vol. 10, no. 3, pp. 630-639, 1992.

[9] G. Bauch, A. F. Naguib, and N. Seshadri, "MAP equalization of space-time coded signals over frequency selective channels," in Proc. Wireless Communications and Networking Conference, New Orleans, La, USA, September 1999.

[10] A. F. Naguib, "Equalization of transmit diversity space-time coded signals," in IEEE Globecom 2000, pp. 1077-1082, San Francisco, Calif, USA, December 1999.

[11] R. Schober, W. H. Gerstacker, and J. B. Huber, "Adaptive linear equalization combined with noncoherent detection for MDPSK signals," IEEE Trans. Communications, vol. 48, no. 5, pp. 733-738, 2000.

[12] F. Edbauer, "Bit error rate of binary and quaternary DPSK signals with multiple differential feedback detection," IEEE Trans. Communications, vol. 40, no. 3, pp. 457-460, 1992.

[13] S. Haykin, Adaptive Filter Theory, Prentice-Hall, Upper Saddle River, NJ, USA, 3rd edition, 1996.

[14] T. Kailath, A. H. Sayed, and B. Hassibi, Linear Estimation, Prentice-Hall, Upper Saddle River, NJ, USA, 2000.

[15] G. L. Stuber, Principles of Mobile Communication, Kluwer Academic, Boston, Mass, USA, 2nd edition, 2001.

[16] Y. Liu and X. Wang, "Multiple-symbol decision-feedback space-time differential decoding in fading channels," EURASIP Journal on Applied Signal Processing, vol. 2002, no. 3, pp. 297-304, 2002, Special Issue on Space-Time Coding and Its Applications-Part I.

Genyuan Wang received his B.S. and M.S. degrees in mathematics from Shaanxi Normal University, Xi'an, China, in 1985 and 1988, respectively, and the Ph.D. degree in electrical engineering from Xidian University, Xi' an China, in 1998. From July, 1988 to September 1994, he worked at Shaanxi Normal University as an Assistant Professor and then an Associate Professor. From September 1994 to May 1998, he worked at

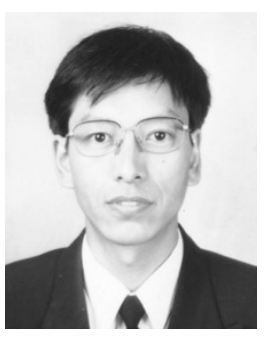
Xidian University as a Research Assistant. Currently, he is PostDoctoral Fellow at the Department of Electrical and Computer Engineering, University of Delaware, USA. His research interests include radar imaging and radar signal processing, adaptive filter, OFDM system, channel equalization, and coding theory.
Aijun Song received his B.S. and M.S. degrees in electrical engineering in Xidian University, Xi'an, China, in 1997 and 2000, respectively. From 1997 to 2000, he was a Research Assistant with National Key Lab for Radar Signal Processing in Xidian University, Xi'an, China. Since 2000, he has been a Research Assistant with the Department of Electrical and Computer Engineering, University of Delaware, USA. His general interests include space-time coding techniques and multicarrier modulations in communications.

Xiang-Gen Xia received his B.S. degree in mathematics from Nanjing Normal University, Nanjing, China, his M.S. degree in mathematics from Nankai University, Tianjin, China, and his Ph.D. degree in electrical engineering from the University of Southern California, Los Angeles, USA in 1983, 1986, and 1992, respectively. He was a Lecturer at Nankai University, China during 1986-1988, a Teaching Assistant at Univer-

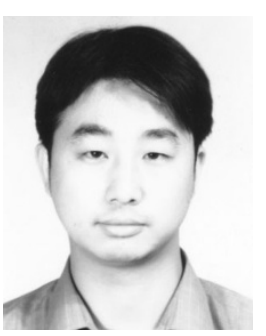
sity of Cincinnati, USA during 1988-1990, a Research Assistant at the University of Southern California, USA during 1990-1992, and a Research Scientist at the Air Force Institute of Technology during 1993-1994. He was a Senior/Research Staff Member at Hughes Research Laboratories, Malibu, California, during 1995-1996. In September 1996, he joined the Department of Electrical and Computer Engineering, University of Delaware, USA, where he is currently an Associate Professor. His current research interests include communication systems including equalization and coding; SAR and ISAR imaging of moving targets, wavelet transform and multirate filterbank theory and applications; time-frequency analysis and synthesis; and numerical analysis and inverse problems in signal/image processing. Dr. Xia has over 80 refereed journal articles published, and 4 U.S. patents awarded. He is the author of the book "Modulated Coding for Intersymbol Interference Channels" (New York, Marcel Dekker, 2000). Dr. Xia received the National Science Foundation (NSF) Faculty Early Career Development (CAREER) Program Award in 1997, the Office of Naval Research (ONR) Young Investigator Award in 1998, and the Outstanding Overseas Young Investigator Award from the National Nature Science Foundation of China in 2001. He also received the Outstanding Junior Faculty Award of the Engineering School of the University of Delaware in 2001. He is currently an Associate Editor of the IEEE Transactions on Mobile Computing, the IEEE Transactions on Signal Processing and the EURASIP Journal on Applied Signal Processing. He is also a member of the Signal Processing for Communications Technical Committee in the IEEE Signal Processing Society. 